\title{
3D Shape Landmark Correspondence by Minimum Description Length and Local Linear Regularization
}

\author{
M. Valenti ${ }^{2,1}$, C. Chen ${ }^{2}$, E. De Momi ${ }^{1}$, G. Ferrigno ${ }^{1}$, and G. Zheng ${ }^{2}$ \\ ${ }^{1}$ Dept. of Electronics, Information and Bioengineering, Politecnico di Milano, Italy \\ 2 Institute for Surgical Technologies and Biomechanics, Universität Bern, Switzerland
}

\begin{abstract}
Statistical Shape Models (SSMs) are currently used in orthopaedic surgery to allow accurate position of prosthetic components through bone morphing and to assess the correct post-operative follow up by virtually reconstructing the surgical site. Focusing on computer assisted Total Knee Arthroplasty (TKA) applications, in this paper we propose a new approach for establishing landmark correspondence of 3D shapes for building SSMs of anatomical structures around the knee joint. Our method is based on the landmark correspondence method by Minimum Description Length (MDL) and enforces local geometric similarity. Our new constraint, which is in the form of local linear regularization, ensures that the local shape geometry of corresponding landmarks on different shapes is similar. We tested our method on building SSMs of three anatomical structures from 24 MRI images of pathological knees, namely femur, patella and tibia. Compared with the original method using only the MDL criterion, our method shows significant improvement in two out of the three structures.
\end{abstract}

Keywords-Computer Assisted Surgery, Image Processing, Statistical Shape Model, Minimum Description Length, Landmark Correspondence.

\section{INTRODUCTION}

In the field of computer vision and medical image processing, statistical shape analysis [2] is an important research tool. Different types of SSMs have been proposed, allowing accurate modelling of shape structure and variation. Most SSMs treat a shape instance as a vector which is built from landmarks. Therefore, to make different shape vectors comparable and to construct a meaningful SSM, it is crucial that the landmarks identified on different shape instances are well corresponded.

There has been a considerable work on automatic landmark correspondence in literature. An earlier work of Brett and Taylor [1] tackled this problem by ICP algorithm. In [9], the landmark sliding algorithm was proposed which features an objective function which encodes both global shape deformation and local shape topology. Xie and Heng [10] developed an algorithm where the shape correspondence is first established by the shape skeleton features and then refined via point matching by a assignment problem.
Recently, methods based on MDL criterion have shown promising results. The MDL criterion was first employed for landmark correspondence in [3], and was shown to generate superior results in [7]. In [4], Heimann et al. proposed a new procedure based on the MDL criterion which is more computationally inexpensive and easier to implement.

In this paper, we propose a new extension to the existing landmark corresponding method. Our method is based on the framework as in [4]. Motivated by the fact that MDL criterion pays more attention on global consistency, we introduce a new constraint that enforces the local shape similarity. Our new constraint, which is based on the local linear regularization, enforces that the local shape geometry is similar on the corresponded landmark on different shapes. By combining the standard MDL criterion with our new constraint, we end up with an objective function which enforces the correspondence from both global and local points of view.

We tested our new algorithm using 24 MRI images of pathological knees, entailing femur, patella and tibia. We use the bipartite matching difference and the Wald-Wolfowitz test [6] as performance measures. We compared our result with the original MDL method and observe improvement of our method in the case of constructing femur and patella SSMs.

\section{THE PROPOSED METHOD}

\section{A. Problem Formulation}

Considering a set of $M$ training shapes, each of which is a triangulated mesh $\left\{S_{m}=\left(V_{m}, E_{m}\right)\right\}_{m=1 \ldots M}, V_{m}$ and $E_{m}$ are the sets of vertices and edges of the $m$ th training shape. Each shape is a Genus Zero Surface, a closed surface that can be parametrized onto the unit sphere. Let us denote $\Omega_{m}\left(S_{m}\right)$ as the spherical parametrization of the $m$ th training shape [8]. For any vertex $v \in V_{m}, \Omega_{m}(v) \in \mathbb{R}^{3}$, where $\left|\Omega_{m}(v)\right|=1$, specifies the coordinate of $v$ on the unit sphere.

A set of $N$ landmarks, $\left\{\left(\tilde{\theta}_{n}, \tilde{\phi}_{n}\right)\right\}_{n=1 \ldots N}$ is also defined on the sphere, where $\left(\tilde{\theta}_{n}, \tilde{\phi}_{n}\right)$ is the spherical coordinates of the $n$th landmark. To calculate the actual position of the $n$th landmark on the $m$ th training shape, we build a ray from the origin to $\left(\tilde{\theta}_{n}, \tilde{\phi}_{n}\right)$ on the parametrization sphere, and calculate the intersection of the ray with the $m$ th shape mesh. We denote $a_{m}^{n}$ 
as the position of the $n$th landmark on the $m$ th training shape.

Our goal is to establish landmark correspondence over the training shapes. The landmarks are defined on the parametrization sphere (i.e. $\left\{\left(\tilde{\theta}_{n}, \tilde{\phi}_{n}\right)\right\}_{n=1 \ldots N}$ is fixed for all shapes). For every training shape $S_{m}$, its parametrization $\Omega_{m}$ solely determines $\left\{a_{m}^{n}\right\}_{n=1 \ldots N}$, the actual position of each landmarks on that shape. Therefore, our goal is searching for the optimal parametrizations $\left\{\Omega_{m}\right\}_{m=1 \ldots M}$ under which the landmark positions over the training shapes have optimal correspondences.

\section{B. Miminum Description Length Cost Function}

Given the set of all landmark positions on all training shapes $\left\{a_{m}^{n}\right\}_{m=1 \ldots M, n=1 \ldots N}$, in [4], the quality of landmark correspondence is defined as:

$$
\begin{gathered}
F_{M D L}=\sum_{k} \mathscr{L}_{m}, \text { where } \\
\mathscr{L}_{m}=\left\{\begin{array}{cc}
1+\log \left(\lambda_{m} / \lambda_{\text {cut }}\right), & \text { if } \lambda_{m} \geq \lambda_{\text {cut }} \\
\lambda_{m} / \lambda_{\text {cut }}, & \text { if } \lambda_{m}<\lambda_{\text {cut }}
\end{array}\right.
\end{gathered}
$$

where $\lambda_{\text {cut }}$ is a parameter which represents the expected noise in the training data and $\lambda_{m}$ is the $m^{\text {th }}$ eigenvalue of the distribution. In [4], the parametrization $\left\{\Omega_{m}\right\}_{m=1 \ldots M}$ is optimized so that the corresponding landmark positions $\left\{a_{m}^{n}\right\}_{m=1 \ldots M, n=1 \ldots N}$ generates the minimum $F_{M D L}$

\section{Local Linear Regularization Cost Function}

In this paper we extend the original MDL-based approach with a new objective function considering the local linear similarity. The idea is to enforce the geometric consistency in the local neighbourhoods of each landmark over different shapes. To do this, we first create a neighbourhood system $\mathscr{N}$ of the landmarks. Specifically, for the $n$th landmark, $\mathscr{N}(n)=$ $\left\{\mathscr{N}(n)_{k}\right\}_{k=1 \ldots K_{n}}$ is the set of $K_{n}$ landmark indices that are within its local neighbourhood. Each neighbour shares with the selected landmark one edge of the landmark shape. Then, considering $a_{m}^{n}$, which is the position of the $n$th landmark on the $m$ th training shape, it should be reasonably reconstructed using its neighbouring landmarks on the same shape:

$$
a_{m}^{n}=w_{m, n}^{1} a_{m}^{\mathscr{N}(n)_{1}}+\ldots+w_{m, n}^{K_{n}} a_{m}^{\mathscr{N}(n)_{K}}=A_{m}^{n} W_{m, n}
$$

where $A_{m}^{n}=\left[a_{m}^{\mathscr{N}(n)_{1}}, \ldots, a_{m}^{\mathscr{N}(n)_{K_{n}}}\right]$ is the matrix of neighbouring landmarks of $a_{m}^{n}$, and $W_{m, n}=\left[w_{m, n}^{1}, \ldots, w_{m, n}^{K_{n}}\right]^{\top} \in \mathbb{R}^{K_{n}}$ is the reconstruction coefficient vector. Note that Eq. (3) only considers reconstructing a single landmark on a single shape. In a usual non-degenerate case, Eq. (3) is underdetermined as long as $K_{n}>3$. However, if we consider the $n$th landmark on every training shape, it is natural to require that the same reconstruction weight is used to reconstruct the same landmark on all shapes. That is, $W_{m, n}$ should be independent of $m$. We thus drop the subscript $m$, and denote the reconstruction weight as $W_{n}$, and Eq. (3) becomes a system of equations defined on all shapes:

$$
\forall m=1, \ldots M: a_{m}^{n} \approx A_{m}^{n} W_{n}
$$

Note that since in our case the number of training shapes is larger than the number of neighbours, Eq. (4) becomes overdetermined and $W_{n}$ can be solved by Least Mean Squares (LMS) method, given that the landmark positions $a_{m}^{n}$ and $A_{m}^{n}$ are known. In this way we can compute $\left\{W_{n}\right\}$ for $n=1 \ldots N$, and then the quality of landmark correspondence $\left(F_{L L R}\right)$ can be expressed as the summation of reconstruction errors of all landmarks over all shapes:

$$
F_{L L R}=\sum_{n=1}^{N} \sum_{m=1}^{M}\left\|a_{m}^{n}-A_{m}^{n} W_{n}\right\|^{2}
$$

Eq.(5) is our objective in terms of the local linear regularization. We add it to the original objective based on MDL, and get the final objective function $(F)$ :

$$
F=F_{M D L}+\alpha F_{L L R}
$$

with $\alpha$ being a positive weighing parameter controlling the relative importance of the new term.

\section{Optimization Process}

To find the parametrizations $\left\{\Omega_{m}\right\}$ that optimize the objective function, we adopt a similar optimization strategy as in [4] and adapt it to our objective function with the new term.

Initialization. For each training shape $S_{m}$, we initialize $\Omega_{m}$ as a conformal parametrization as in [8].

Iterative Optimization. We iteratively optimize the set of parametrizations $\left\{\Omega_{m}\right\}$. In each iteration, for each shape, we locally update the parametrization using an update function $\Omega^{\prime}=\Phi(\Omega)$ which is parametrized as $\Omega^{\prime}=\Phi_{c, \sigma, \Delta \theta, \Delta \phi}(\Omega)$, where $c$ and $\sigma$ are the centre and bandwidth of the update kernel, and $\Delta \theta$ and $\Delta \phi$ specify the update direction. We use a Gaussian envelope kernel:

$\rho(x)=\left\{\begin{array}{cc}\exp \left(\frac{-\|x-c\|^{2}}{2 \sigma^{2}}\right)-\exp \left(\frac{-(3 \sigma)^{2}}{2 \sigma^{2}}\right) & \text { for }\|x-c\|<3 \sigma \\ 0 & \text { for }\|x-c\| \geq 3 \sigma\end{array}\right.$ 
Eq. (7) gives the magnitude of change at any point $x$ on the mesh. Combined with the direction of update, we actually change the spherical coordinate of $x$ by $\rho(x) \times(\Delta \theta, \Delta \phi)$.

The update direction $(\Delta \theta, \Delta \phi)$ in each iteration is determined through the gradient of the objective function with regard to $(\Delta \theta, \Delta \phi)$. Since the influence of $(\Delta \theta, \Delta \phi)$ to the objective $F$ is via the landmark positions we have:

$$
\frac{\partial F}{\partial(\Delta \theta, \Delta \phi)}=\frac{\partial F}{\partial a_{i}^{j}} \frac{\partial a_{i}^{j}}{\partial(\Delta \theta, \Delta \phi)}
$$

where $\frac{\partial a_{i}^{j}}{\partial(\Delta \theta, \Delta \phi)}$ is calculated by finite difference method. $\frac{\partial F}{\partial a_{i}^{j}}$ is calculated analytically. From Eq. (6), we have:

$$
\frac{\partial F}{\partial a_{i}^{j}}=\frac{\partial F_{M D L}}{\partial a_{i}^{j}}+\alpha \frac{\partial F_{L L R}}{\partial a_{i}^{j}}
$$

where $\frac{\partial F_{M D L}}{\partial a_{i}^{j}}$ is calculated as in [4]. For $\frac{\partial F_{L L R}}{\partial a_{i}^{j}}$, note from Eq.(5) that in each component of summation $\left\|a_{m}^{n}-A_{m}^{n} W_{n}\right\|^{2}$, depending on the relation of $(m, n)$ and $(i, j), a_{i}^{j}$ might not appear, or might appear in $a_{m}^{n}$ or $A_{m}^{n}$ (but not both). Therefore:

$$
\begin{gathered}
\frac{\partial F_{L L R}}{\partial a_{i}^{j}}=\sum_{m=1}^{M} \sum_{n=1}^{N} f_{(m, n)}^{\prime}(i, j), \text { where } \\
f_{(m, n)}^{\prime}(i, j)=\left\{\begin{array}{cc}
2 a_{i}^{j}-2 W_{j} A_{i}^{j} & \text { if }(m, n)=(i, j) \\
2 W_{n}^{d}\left(W_{n} A_{i}^{n}-a_{i}^{n}\right) & \text { if } m=i \text { and } j=\mathscr{N}(n)_{d} \\
0 & \text { otherwise }
\end{array}\right.
\end{gathered}
$$

\section{EXPERIMENTS}

In order to evaluate the performances of our new method, we used a dataset of 24 MRI images of pathological knees. The images were manually segmented using Amira ${ }^{\circledR}$ (VSG3D, France), then reduced and rigidly aligned. We then used both methods to register three different groups of anatomical structures, namely femurs, patellas and tibias. Table 1 shows the number of points of each mesh and the number of landmarks used for each group.

Table 1 Schema of the three different experiments done

\begin{tabular}{clcc}
\hline Group & Anatomical part & \# points & \# landmarks \\
\hline 1 & Femur & 5002 & 2562 \\
2 & Patella & 5002 & 642 \\
3 & Tibia & 5002 & 2562 \\
\hline
\end{tabular}

For each group, we ran the algorithm with $\alpha$ equal to 0 (for the original algorithm) and with $\alpha=0.5$ representing our new implementation (cfr. Eq.(6)). To evaluate the performance of the two algorithms we used the coefficient described in [6] which states that the well known measurements of compactness, specificity and generalization could have some limitation in the evaluation of a statistical shape model. In [6] Munsell et al. describe a new benchmark for the evaluation of $2 \mathrm{D}$ shape-space based on a given ground truth. We extended this method to 3D volume-space, and used the original shapes as ground truth. Formally, the evaluation of the shape correspondence follows these steps:

- each shape resulting from the two algorithms $\left(\left\{S_{i}^{r}\right\}_{i=1}^{M}\right)$ is rigidly realigned with its original shape $\left(\left\{S_{i}^{o}\right\}_{i=1}^{M}\right)$

- each shape, including the ground-truth shapes, is then voxelized with a grid of $0.5 \times 0.5 \times 0.5 \mathrm{~mm}$.

At this point we need to introduce the Jaccard coefficient. This is defined as

$$
\Delta\left(S_{1}, S_{2}\right)=1-\frac{\left|R\left(S_{1}\right) \cap R\left(S_{2}\right)\right|}{\left|R\left(S_{1}\right) \cup R\left(S_{2}\right)\right|}
$$

where $S_{1}, S_{2}$ are the two shapes considered and $|R|$ computes the volume enclosed in the surface.

\section{A. Bipartite Matching Difference Measure}

The first measure we define is based on the bipartitematching difference between $\left\{S_{i}^{o}\right\}_{i=1}^{M}$ and $\left\{S_{j}^{r}\right\}_{j=1}^{M}$. We build up the graph that has $2 M$ vertices for the shapes $\left\{S_{i}^{o}\right\}_{i=1}^{M}$ and $\left\{S_{j}^{r}\right\}_{j=1}^{M}$. The weight of the link between two different shapes is given by the Jaccard coefficient between the two shapes linked. Then, applying the bipartite matching algorithm (with the Hungarian method [5]), we can match each ground truth shape with each result shape in order to minimize the sum of the weights. The bipartite matching difference measure is defined as

$$
\Delta_{b}=\frac{\sum_{i=1}^{M} \Delta\left(S_{i}^{o}, S_{b(i)}^{r}\right)}{M}
$$

where $\Delta\left(S_{i}^{o}, S_{b(i)}^{r}\right)$ is the Jaccard difference of the identified corresponding shapes. Thanks to the normalization, $\Delta_{b}$ is always a value in $[0,1] ; \Delta_{b}=0$ implies that the two shape space compared come from the same distribution, while if $\Delta_{b}=1$ they describe two completely different shape spaces.

\section{B. Wald-Wolfowitz Test}

The second measure we take into consideration is the Wald-Wolfowitz generalized test, based on the minimum 
spanning tree (MST) algorithm. For this algorithm, we build a fully connected undirected graph with $2 \mathrm{M}$ vertices, that represent both $\left\{S_{i}^{o}\right\}_{i=1}^{M}$ and $\left\{S_{i}^{r}\right\}_{i=1}^{M}$. Then we define the weight of each edge connecting two shapes (both inter and intra the two spaces) as the Jaccard coefficient between the two shapes. We then find the MST of the constructed graph, that is the spanning tree with the minimum total edge weight. On this tree, we count the number of edges that connect two shapes from the same space, either inside $\left\{S_{i}^{o}\right\}_{i=1}^{M}$ or $\left\{S_{i}^{r}\right\}_{i=1}^{M}$. We can call this number $W$. Normalizing $W$ over $2 M-2$ we finally get the Wald-Wolfowitz difference measure $\left(\Delta_{w}\right)$.

The $\Delta_{w}$ value is thus always included in $[0,1]$. In particular, a smaller value of $\Delta_{w}$ indicates that the two distributions most likely come from the same shape space.

\section{Results}

We evaluate the performances of the two algorithms both with the two methods described above and with a visual/qualitative comparison.

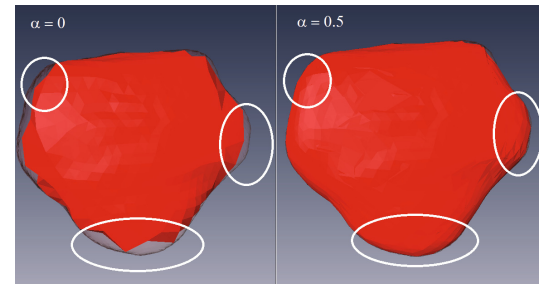

Fig. 1 Same patella mesh processed with two different values of $\alpha$

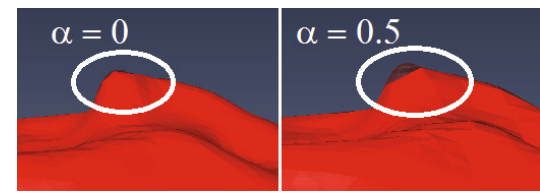

Fig. 2 Same tibia mesh processed with two different values of $\alpha$

Qualitative differences between the two models are highlighted in Figure 1 and 2. For the quantitative results, Table 2 describes the two index achieved with $\alpha=0$ (as in [4]) and $\alpha=0.5$ (the present algorithm) for the investigated bones.

\section{Discussion}

Quantitative results show that our method performs better in case of femur and patella, while no improvement is made in case of tibia. Evaluating visually the performances, we can see that tibia has some sharp contours that cannot be rightly approximated by our algorithm, based on the similarity of neighbouring points. However, such low levels of $\Delta_{b}$
Table 2 Schema of the results achieved

\begin{tabular}{cccc}
\hline Anatomical part & index & $\alpha=0$ & $\alpha=0.5$ \\
\hline \multirow{2}{*}{ Femur } & $\Delta_{b}$ & 0.0226 & 0.0204 \\
& $\Delta_{w}$ & 0.4348 & 0.3913 \\
\hline \multirow{2}{*}{ Patella } & $\Delta_{b}$ & 0.0786 & 0.0449 \\
& $\Delta_{w}$ & 0.500 & 0.4783 \\
\hline \multirow{2}{*}{ Tibia } & $\Delta_{b}$ & 0.0108 & 0.0117 \\
& $\Delta_{w}$ & 0.4565 & 0.500 \\
\hline
\end{tabular}

achieved for tibia with both algorithms, indicates that the two shape space contain similar meshes and also that a shape has the same probability density in these two shape spaces.

\section{CONCLUSIONS}

We describe a new algorithm to improve landmark correspondences on different shapes for statistical shape analysis. We evaluate our new method with two different quantitative measures and with a qualitative overview of the results.

While our method is better in two cases (femur and patella) out of three, we cannot state that for every shape we can achieve better results. Further investigation will be done on other bones, in order to asses the improvement given by our landmark correspondences optimization method.

\section{REFERENCES}

1. A.D. Brett, C.J. Taylor. A method of automated landmark generation for automated 3D PDM construction. Image and Vision Computing 18 (2000) 739-748.

2. T.F. Cootes, C.J. Taylor. Active shape models - smart snakes. In: BMVC (1992).

3. R.H. Davies, C.J. Twining, T.F. Cootes, J.C. Waterton, C.J. Taylor. 3D statistical shape models using direct optimisation of description length. In: ECCV (2002).

4. T. Heimann, I. Wolf, T. Williams, H.-P. Meinzer. 3D active shape models using gradient descent optimization of description length. In: IPMI (2005).

5. H.W. Kuhn. The Hungarian method for the assignment problem. Naval Research Logistics (1955).

6. B.C. Munsell, P. Dalal, S.Wang. Evaluating Shape Correspondence for Statistical Shape Analysis: A Benchmark Study. In: IEEE Pattern Analysis and Machine Intelligence (2008)

7. M. Styner, K.T. Rajamani, L.P. Nolte, G. Zsemlye, G. Szekely, C.J. Taylor, R.H. Davies. Evaluation of 3D correspondence methods for model building. In: IPMI (2003).

8. H.H. Thodberg. Minimum description length shape and appearance models. In: IPMI (2003).

9. S. Wang, T. Kubota, T. Richardson. Shape correspondence through landmark sliding. In: CVPR (2004).

10. J. Xie, P. Heng. Shape modeling using automatic landmarking. In: MICCAI (2005). 